\title{
First assessment of new Evaluated Data Libraries for Monte Carlo particle transport
}

\author{
Maria Grazia Pia*† \\ INFN Sezione di Genova \\ E-mail: mariagrazia.pia@ge.infn.it \\ Tullio Basaglia \\ CERN \\ E-mail: tullio.basaglia@cern.ch \\ Matteo Bonanomil \\ Università di Milano Bicocca \\ E-mail: m.bonanomi7@campus.unimib.it

\section{Federico Cattorini} \\ Università di Milano Bicocca \\ E-mail: f.cattorini@campus.unimib.it

\section{Chansoo Choi} \\ Hanyang University \\ E-mail: cchoi91@hanyang.ac.kr \\ Min Cheol Han \\ INFN Sezione di Genova \\ E-mail: mchan@hanyang.ac.kr
}

\section{Gabriela Hoff}

Università di Cagliari and INFN Sezione di Cagliari

E-mail: ghoff.gesic@gmail.com

\section{Chan Hyeong Kim}

Hanyang University

E-mail: chkim@hanyang.ac.kr

\section{Sung Hun Kim}

Hanyang University

E-mail: ksh4249@hanyang.ac.kr

\section{Matteo Marcoli}

Università di Milano Bicocca

E-mail: m.marcoli@campus.unimib.it

\section{Paolo Saracco}

INFN Sezione di Genova

E-mail: paolo.saraccolge. infn.it 
Evaluated data libraries are the foundation of physics modeling in Monte Carlo particle transport codes, such as Geant4, FLUKA and MCNP, which are used in high energy and nuclear physics experiments, accelerator studies and detector development. They encompass recommended cross sections, nuclear and atomic parameters, which may derive from theoretical calculations, evaluations of experimental data or a combination of both. New versions of major, widely used evaluated data libraries were released in early 2018 by the IAEA (International Atomic Energy Agency) and the NNDC (National Nuclear Data Center, BNL); among them, the new data libraries for electron-photon transport represent substantial evolutions with respect to those currently in use, which date back to more than 20 years ago. The changes concern both the physics content and the data structure, which in turn affect the physical the reliability and the computational performance of simulations. The main features of the new data libraries are summarized, with emphasis on what has changed, along a first assessment of their physics quality and of their effects on computational performance in the Geant 4 environment. These results provide quantitative and objective elements for developers and users of Monte Carlo codes to evaluate the impact of migrating simulations to new data libraries on sound grounds. The assessment also highlights opportunities for improvement in future releases.

The 39th International Conference on High Energy Physics (ICHEP2018)

4-11 July, 2018

Seoul, Korea

\footnotetext{
* Speaker.

${ }^{\dagger}$ The authors thank the CERN Library for providing valuable material.
} 


\section{Overview}

Evaluated data libraries play a fundamental role in Monte Carlo particle transport. ENDF/BVIII.0 [1] includes new photo-atomic and electro-atomic interaction data, and atomic relaxation data taken from EPICS2017 [2, 3, 4]. The atomic ENDF/B components are based on three data libraries, EPDL (Evaluated Photon Data Library) [5], EEDL (Evaluated Electron Data Library) [6] and EADL (Evaluated Atomic Data Library) [7].

The major change in EPICS2017 concerns EADL atomic binding energies. The new data derive from two compilations, which produce more accurate results than the original EADL in the test scenarios documented in Ref. [8]. Differences are also present in the total cross sections of photon coherent scattering, electron kinetic energies and EEDL excitation data. Very small differences between EPICS2017 and previous releases concerning other data sets can be attributed to roundoff errors.

According to Ref. [3] and [4], the number of data tabulated in the electron and photon data libraries has been increased for the data to be linearly interpolated; nevertheless, not all the data are tabulated with a larger number of energy points.

\section{Assessment the new releases}

Inconsistencies related to the modification of atomic binding energies have been observed: in some cases they could result in energy non-conservation in Monte Carlo particle transport. Inconsistencies are also present in the spectra of secondary electrons generated by electron ionisation. Shortcomings of the transition probability values tabulated in EADL are extensively documented in [9] and [10].

Shortcomings in the configuration management of the data libraries released in 2018 have been observed; they affect version control in the experimental application that use them and, consequently, the reproducibility of results, which is an essential requirement of the scientific method.

The increase of the size of tabulated data to allow their linear interpolation affects the computational performance of the software systems that use the new data libraries. The effects are documented in [10].

Validation tests concerning the previous versions of EEDL, EPDL and EADL are documented in the literature $[8,9,11,12,13,14]$. Those that concern data sets not affected by the modification of atomic binding energies are still pertinent.

In a validation test [15] of the electron impact cross sections for $\mathrm{K}$ and $\mathrm{L}$ shells no statistically significant differences were observed regarding the compatibility with experiment of cross sections based on the old EEDL data and the new EPICS2017 data.

\section{Conclusion}

The new evaluated atomic data libraries released in EPICS2017 and ENDF/B-VIII.0, represent a major evolution in the field, since these libraries are widely used by several Monte Carlo systems for electron and photon transport. 
The compilations of binding energies used to produce the new data libraries were identified as more accurate than the previous EADL values. Nevertheless, inconsistencies are observed in the propagation of the modified binding energies to dependent quantities tabulated in the data libraries, which could be the source of disruptive effects in Monte Carlo simulations.

Concerns are also raised by the lack of proper configuration management and version control, which hinders the reproducibility of simulation results based on the data libraries.

The assessment of the new evaluated atomic data libraries is extensively documented in [10].

\section{References}

[1] D. A. Brown et al., ENDF/B-VIII.0: The 8th Major Release of the Nuclear Reaction Data Library with CIELO-project Cross Sections, New Standards and Thermal Scattering Data, Nucl. Data Sheets 148, 1-142 (2018).

[2] D. E. Cullen, A Survey of Atomic Binding Energies for use in EPICS2017, IAEA-NDS-0224 Document, Vienna, Austria (2017).

[3] D. E. Cullen, A Survey of Photon Cross Section Data for use in EPICS2017, rev. 1, IAEA-NDS-0225 Document, Vienna, Austria (2017).

[4] D. E. cullen, A Survey of Electron Cross Section Data for use in EPICS2017, IAEA-NDS-0226 Document, Vienna, Austria (2017).

[5] D. E. Cullen et al., EPDL97, the Evaluated Photon Data Library, UCRL-50400, Vol. 6, Rev. 5 Report, Lawrence Livermore National Laboratory (1997).

[6] S. T. Perkins et al., Tables and Graphs of Electron-Interaction Cross Sections from $10 \mathrm{ev}$ to $100 \mathrm{GeV}$ Derived from the LLNL Evaluated Electron Data Library (EEDL), UCRL-50400 Vol. 31 Report, Lawrence Livermore National Laboratory (1991).

[7] S. T. Perkins et al., Tables and Graphs of Atomic Subshell and Relaxation Data Derived from the LLNL Evaluated Atomic Data Library (EADL) Z=1-100, UCRL-50400 Vol. 30, Lawrence Livermore National Laboratory (1991).

[8] M. G. Pia et al., Evaluation of atomic electron binding energies for Monte Carlo particle transport, IEEE Trans. Nucl. Sci. 58, 6, 3246-3268 (2011).

[9] M. G. Pia et al., Validation of $K$ and $L$ Shell Radiative Transition Probability Calculations, IEEE Trans. Nucl. Sci. 56, 6, 3650-3661 (2009).

[10] M. C. Han et al., First Assessment of ENDF/B-VIII and EPICS Atomic Data Libraries, IEEE Trans. Nucl. Sci. 65 2268-278, 2018.

[11] M. Batic et al., Photon elastic scattering simulation: validation and improvements to Geant4, IEEE Trans. Nucl. Sci. 59, 4, 1636-1664 (2012).

[12] M. C. Han et al., Validation of cross sections for Monte Carlo simulation of the photoelectric effect, IEEE Trans. Nucl. Sci. 63, 2, pp. 1117-1146, 2016.

[13] H. Seo et al., Ionization cross sections for low energy electron transport, IEEE Trans. Nucl. Sci 58, 6 , pp. 3219-3245, 2011.

[14] S. Guatelli et al., Validation of Geant4 Atomic Relaxation against the NIST Physical Reference Data, IEEE Trans. Nucl. Sci. 54, 3, 594-603 (2007).

[15] T. Basaglia et al., Validation of Shell Ionization Cross Sections for Monte Carlo Electron Transport, IEEE Trans. Nucl. Sci. 65, 2279-2302, 2018. 